\title{
A rescue method for a large femoral trial head trapped into the cup during direct anterior total hip arthroplasty without using a fracture table.
}

\author{
Wei-Jen Chen ${ }^{1 \#}$, Chang-Han Chuang ${ }^{1 \#}$, Chun Hsien Huang ${ }^{1}$, Ming-Chou Ku ${ }^{1,2}$ \\ ${ }^{1}$ Department of Orthopedics, Show Chwan Memorial Hospital, Changhua, Taiwan \\ ${ }^{2}$ Show Chwan Health System, Changhua, Taiwan \\ \#These authors have equally contributed to this study.
}

\begin{abstract}
Minimal invasive direct anterior approach (DAA) is an established procedure for total hip arthroplasty (THA). THA via DAA with a larger head size has been reported to reduce the risk of postoperative dislocation. Additionally, variations of the technique exist among different surgeons. Many surgeons prefer a standard surgical table to a fracture table because a standard surgical table less interfere the use of fluoroscopy and facilitates measuring leg length. Here, we presented a rare complication during THA via DAA with a $36 \mathrm{~mm}$ large head using a standard surgical table. A 59-y-old woman was diagnosed of bilateral dysplastic hip. The first (right) THA via DAA was done smoothly but a significant postoperative leg length discrepancy (LLD) was noted. To precisely evaluate the LLD in the second (left) THA via DAA, we used a standard surgical table. Intraoperatively, during the last trial reduction, the 36 mm trial head was trapped into the cup and could not be re-dislocated. In the meantime, the surgical table is not helpful for anterior traction to dislocate the head. Then, we decided to chisel the trial head and perform a controlled cracking on the calcar using an osteotome for further sub-siding the stem. Finally, the THA was completed smoothly and the patient did not complain a subjective LLD anymore. We suggest that surgeons should be aware of the slightly different length between the trial stem and the real stem, and should be careful about the selection of head size and type of surgical table in THA via DAA.
\end{abstract}

Keywords: Total hip arthroplasty, THA, Rescue in surgery, Head size, Surgical trouble.

Accepted on June 21, 2018

\section{Introduction}

Minimal invasive direct anterior approach (DAA) for total hip arthroplasty (THA) provides several benefits for patients, including muscle sparing and faster functional recovery [1]. However, this approach is technically demanding and usually requires a longer learning curve [2,3]. Variations of the technique exist among different surgeons4. For example, many surgeons prefer a fracture table with perineal post [4,5], while some consider the perineal post unnecessarily interfere the use of fluoroscopy $[4,6,7]$. Additionally, head size has been noticed of its influence on the risk of postoperative dislocation $[8,9]$. Hoell et al. reported that THA via DAA in combination with larger femoral head could significantly reduce the rate of postoperative dislocation [8]. Here, we presented a rare complication during THA via DAA using a $36 \mathrm{~mm}$ large head and a standard surgical table. We also demonstrated a method for rescuing the intraoperative complication.

\section{Case Presentation}

A 59-y-old woman with body mass index of 23.9 was diagnosed of bilateral hip dysplasia. She underwent right THA via DAA in 2016. During the right THA, the smallest (No. 0) $\mathrm{M} / \mathrm{L}$ type cement less femoral stem (United Orthopedic Corporation, Taipei, Taiwan) was used due to the narrow femoral canal. The acetabulum was reamed to $54 \mathrm{~mm}$ and a 54 cement less cup was fixed with two screws under fluoroscopic control. A femoral head made of zirconia ceramic (CeramTec, Plochingen, and Germany) with a $36 \mathrm{~mm}$ cross-linked polyethylene liner was inserted. The course of right THA was smooth without any complications. Postoperatively, however, the patient's right hip was slightly right leg was shorter than left one with $3.62 \mathrm{~mm}$ compared to the un-operated left hip (Figure 1) and she expected the leg length discrepancy (LLD) could be minimized after the left THR. To facilitate evaluating the LLD, the left THA via DAA was performed with the use of a standard surgical table. The hip was centered at the break of the table and elevated with a sand bag. The operated leg was placed on a surgical instrument trolley to facilitate leg length 
comparison. DAA was performed and the capsule was opened. The acetabulum was reamed up to $52 \mathrm{~mm}$ and a $52 \mathrm{~mm}$ cup was impacted with 2 screws under fluoroscopic control. A cross-linked polyethylene cup of $36 \mathrm{~mm}$ liner was impacted in. The left femoral canal was even narrower than the right femur where the smallest stem was used. Only one round of broaching was necessary and the smallest rasp was selected for preparing the femoral canal. A trial head with a short neck on an interposition component was connected to the rasp. Trial reduction was done smoothly. The smallest femoral stem was decided (No. 0), but the trial stem slightly protruded from the cut neck. Initially, the tension of surrounding soft tissue was accepted. Then a $36 \mathrm{~mm}$ trial head with a short neck was used for the last trial. Unfortunately, we found it was unable to redislocate the trial head, which was trapped into the cup. All efforts were made to re-dislocate the trial head but in vain, including bending the surgical table at the hip level to facilitate the traction manoeuvre, releasing the tight capsule, using bone hook to loop the neck and pull it upwardly.

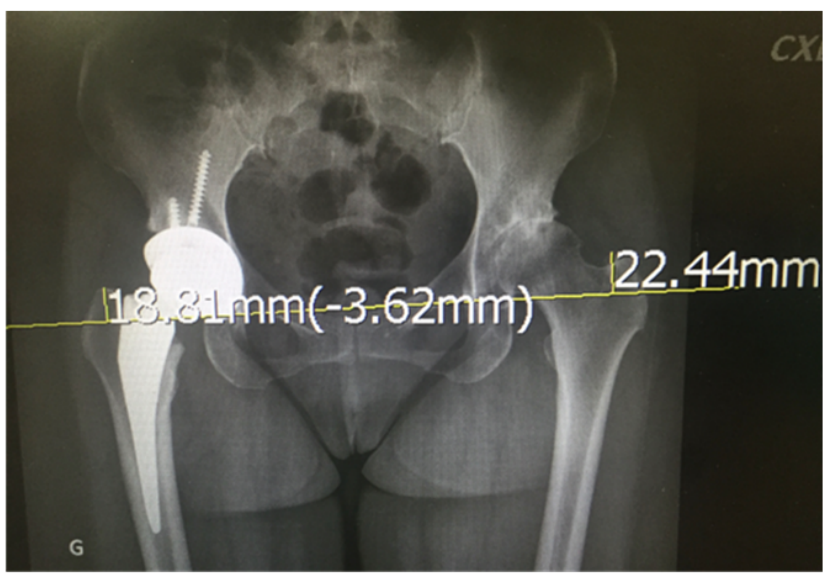

Figure 1. Leg length discrepancy of $3.6 \mathrm{~mm}$ was measured on preoperative $X$-ray.

After 20-min failed efforts, we decided to break the trial head by chisel to rescue the head from the cup. A narrow chisel was selected to prevent damaging the implanted cross-linked polyethylene liner. Since the trial head is made of high density plastic material and is hard and brittle, it was not difficult to break the head. The trial head was rotated 60 degree each time and was chiselled (Figure 2A). At the last hit, the zenith of the trial head was broken and the trunnion was escaped from the liner (Figure 2B). It took about $10 \mathrm{~min}$ to break and redislocate the trial head. Since the femoral stem was the smallest and had been inserted into the femoral canal tightly, it was difficult to further sub-side the stem.

A controlled cracking was performed on the calcar using an osteotome to widen the proximal femur. Then the stem was driven further into the canal, resulting in a $0.5-\mathrm{cm}$ subsidence of the stem. A $36 \mathrm{~mm}$ head with short neck was impacted and reduced. After fluoroscopic confirmation of the stem position and leg length measurement, the surgery was finished with an hour longer than it was expected. The total blood was $850 \mathrm{ml}$ and no blood transfusion was given. The patient stood the surgical procedure well and the postoperative course was smooth without any complications.
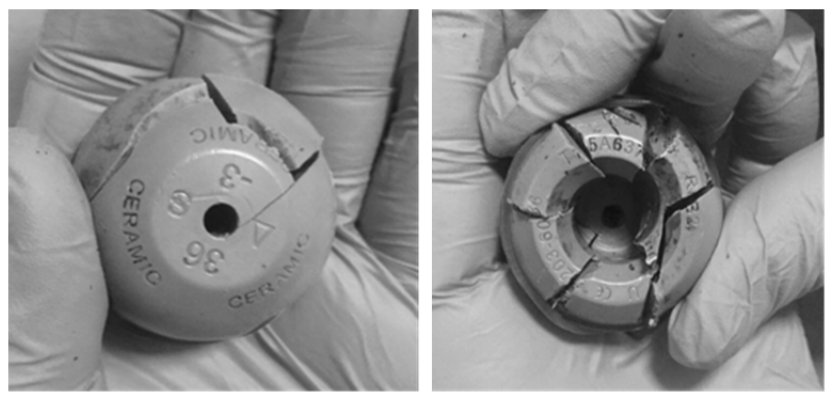

Figure 2. (A) The trial head was rotated 60 degree each time and was chiselled; (B) The zenith of the trial head was broken by the last hit.

The patient resumed mobility with walking assistance on the first postoperative day and was discharged on the 4th postoperative day. The postoperative LLD was corrected to 1 mm (Figure 3) and the patient did not complain a subjective LLD anymore.

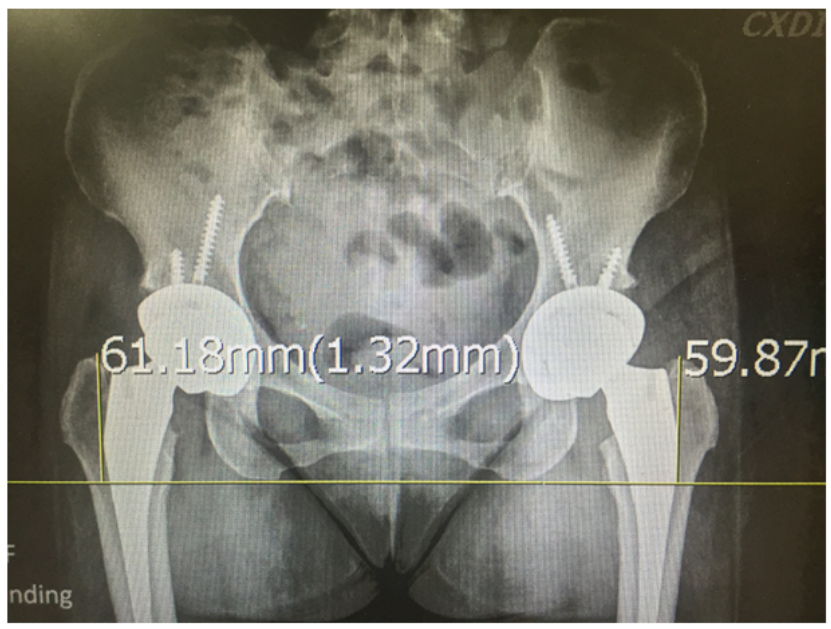

Figure 3. The postoperative leg length discrepancy was corrected to 1 $\mathrm{mm}$.

\section{Discussion}

As a minimal invasive surgery, THA via DAA is an established procedure. It has been reported that THA via DAA with a head size $\geq 36 \mathrm{~mm}$ is associated with good functional recovery and reduced risk of postoperative dislocation. Comparing to a 32 $\mathrm{mm}$ head with an only $16 \mathrm{~mm}$ jump height, a $36 \mathrm{~mm}$ head has an $18 \mathrm{~mm}$ jump height and consequently could reduce dislocation rate significantly. However, a larger head might also increase the difficulties of re-dislocating the trial head. In this case study, we encountered a rare intraoperative complication. We chose a larger head of $36 \mathrm{~mm}$, but unfortunately the trial head was trapped into the cup during the last trial reduction. This complication may not be specific for DAA. It might occur during other approaches with a larger head or during bipolar endoprosthesis.

In posterior approach or in anterior-lateral approach, pelvis is positioned in lateral decubitus position. It is easier to re- 


\section{without using a fracture table}

dislocate the trial head because the force of traction is mainly distal and posterior in direction which can be facilitated by leveraging the lower leg and rotating the femur. In DAA, the pelvis is put in supine and the force to dislocate the trial head is applied distally and anteriorly. Thus, anterior traction is against the gravity and is laborious and less efficient. In this situation, a fracture table with a perineal post or a dedicated traction table for DAA would be helpful for anterior traction to dislocate the head.

However, some surgeons prefer a standard surgical table than a fracture table because of some advantages. A standard surgical table would interfere less the use of fluoroscopy and it is easier to measure hip stability, range of motion, and leg lengths. To precisely measure the leg lengths was our major concern in this case. Thus, we chose to use a standard surgical table. Unfortunately, the surgical table was not helpful while the trial head trapped into the cup.

According to our experience, we suggest that surgeons should be aware of the slightly different length between the trial stem and the real stem and should be careful about the selection of head size and type of surgical table.

\section{References}

1. Barnett SL, Peters DJ, Hamilton WG. Is the anterior approach safe? Early complication rate associated with 5090 consecutive primary total hip arthroplasty procedures performed using the anterior approach. J Arthroplasty 2016; 31: 2291-2294.

2. De Steiger RN, Lorimer M, Solomon M. What is the learning curve for the anterior approach for total hip arthroplasty. Clin Orthop Relat Res 2015; 473: 3860-3866.

3. Spaans AJ, van den Hout JA, Bolder SB. High complication rate in the early experience of minimally invasive total hip arthroplasty by the direct anterior approach. Acta Orthop 2012; 83: 342-346.

4. Connolly KP, Kamath AF. Direct anterior total hip arthroplasty: Literature review of variations in surgical technique. World J Orthop 2016; 7: 38-43.

5. Jewett BA, Collis DK. High complication rate with anterior total hip arthroplasties on a fracture table. Clin Orthop Relat Res 2011; 469: 503-507.

6. Lovell TP. Single-incision direct anterior approach for total hip arthroplasty using a standard operating table. J Arthroplasty 2008; 23: 64-68.

7. Oinuma K, Tamaki T, Miura Y. Total hip arthroplasty with sub-trochanteric shortening osteotomy for Crowe grade 4 dysplasia using the direct anterior approach. J Arthroplasty 2014; 29: 626-629.

8. Hoell S, Sander M, Gosheger G. The minimal invasive direct anterior approach in combination with large heads in total hip arthroplasty - is dislocation still a major issue? A case control study. BMC Musculoskelet Disord 2014; 15: 80 .

9. Cooper HJ, Della Valle CJ. Large diameter femoral heads: is bigger always better? Bone Joint J 2014; 96: 23-26.

\section{*Correspondence to}

Chang-Han Chuang

Department of Orthopedics

Show Chwan Memorial Hospital

Changhua

Taiwan 